\title{
Linealización de Amplificadores de Potencia RF mediante Transformada Wavelet
}

\author{
Carlos Mateo, Pedro L. Carro, Paloma García, Jesús de Mingo y José Ramón Pérez \\ Grupo de Comunicaciones Móviles (GCM) \\ Instituto de Investigación en Ingeniería de Aragón (I3A) \\ Universidad de Zaragoza, Mariano Esquillor s/n, 50018, Zaragoza, Spain. \\ Tel. +34-976762707, e-mail: cmperez@unizar.es
}

\begin{abstract}
Se presenta una nueva técnica para el modelado de amplificadores de potencia de banda ancha (AP) y diseño de predistorsionadores digitales (DPD). Dicha técnica consiste en descomponer la señal original en niveles multirresolución mediante la Transformada Wavelet. Se evaluará en un sistema de Radio Cognitiva WRAN (IEEE 802.22).
\end{abstract}

\section{Introducción}

En los sistemas de comunicaciones actuales se utilizan esquemas multiportadora (OFDMA) con modulaciones de alta eficiencia espectral (PSK, QAM), caracterizadas por no tener envolvente constante y alto valor de PAPR. Esto hace que la señal se deforme al pasar por elementos no lineales, como lo son los amplificadores de potencia AP. Además esto produce un recrecimiento espectral en las bandas de frecuencia adyacentes (ACI). El objetivo es reducir esta distorsión sin repercutir en la eficiencia energética.

Una de las mejores opciones es el uso de predistorsionadores digitales (DPD), los cuales generan una distorsión complementaria a la del AP. Sin embargo, la ganancia total del sistema (AP + DPD) puede ser inferior a la del propio AP, disminuyendo la ganancia en la banda útil.

Tanto para modelar el PA como para diseñar el DPD a lo largo de la literatura se han utilizado versiones simplificadas de las series de Volterra [1], pero no son capaces de adaptarse a las condiciones cambiantes de la transmisión en sistemas de Radio Cognitiva. La técnica propuesta tratará de mejorar la identificación de los coeficientes tanto del AP como del DPD en sistemas con condiciones de transmisión cambiantes.

\section{Modelo propuesto}

El modelo de Multirresolución propuesto consiste en descomponer la señal en varios niveles multirresolución [2] como

$$
x(n)=c(n)_{J}+\sum_{j=1}^{J} d(n)_{j},
$$

siendo $x(n)$ la señal de entrada, $c(n)_{J}$ su aproximación y $d(n)_{J}$ sus detalles. Cada nivel es obtenido de la descomposición de la aproximación del nivel anterior. El análisis multirresolución (MR) es llevado a cabo por la Stationary Wavelet Transformation (SWT), la cual mantiene el número de muestras en cada nivel. El proceso MR está basado en la función wavelet Daubechies [3], la cual asegura una independencia entre la información en la aproximación y los detalles. El proceso se hará hasta el nivel de descomposición $J$, obteniendo la aproximación de nivel $J$, los detalles del mismo nivel y del $J-1$. Solamente se utilizarán dos niveles de resolución para no aumentar el coste computacional en exceso.

Los parámetros de no linealidad $N$, de profundidad de memoria $M$ y nivel de resolución $J$ se han elegido mediante el método BIC, y el orden de la wavelet el cual minimiza el error producido.

\section{Setup de medida}

El montaje consiste en un PC con Matlab, generador de señales Agilent E4438C, osciloscopio Agilent Infiniium DSA90804A. El dispositivo bajo test un amplificador ZHL-4240 de Minicircuits con una ganancia de de $40 \mathrm{~dB}$ a $774 \mathrm{MHz}$. Se ha trabajado sobre el estándar WRAN con señales OFDMA 16QAM tanto para el enlace de subida como para el de bajada con un ancho de banda de $16 \mathrm{MHz}$.

\section{Resultados}

Los resultados del modelado del AP y del DPD han sido evaluados en base al NMSE, la mejora de la $A C I$ en base a la relación $\left(\mathrm{R}_{\mathrm{pot}}\right)$, que se define

$$
R_{\text {pot }}=10 \log _{10}\left(\frac{P_{\text {adjch }}}{P_{\text {adjch }}}\right)
$$


Para conocer los cambios en las condiciones de transmisión se calcula la coherencia entre la señal de entrada y la de salida linealizada, definida como

$$
\gamma(t, f)=\frac{\left|S_{x y_{l i n}}(t, f)\right|}{\sqrt{S_{x x}(t, f) S_{y_{l i n} y_{l i n}}(t, f)}}
$$

donde $S_{x y l i n}$ es la correlación entre la señal de entrada y la de salida linealizada, $S_{x x}$ y $S_{y y}$ la autocorrelación de ambas respectivamente.

En la Tabla 1 aparecen los resultados obtenidos tanto para el modelo clásico polinomial (P) como para el modelo propuesto de multirresolución (M). Para ambos los valores de los parámetros son $N=7$ y $M=5$; mientras que para el segundo $J=2$.

Tabla 1. Resultados obtenidos con predistorsionadores Polinomial (P) y Multirresolución (M).

\begin{tabular}{|c|c|c|c|c|}
\hline Señal & Modelo & $\begin{array}{c}\text { Orden } \\
\text { wavelet }\end{array}$ & $\begin{array}{c}\text { NMSE } \\
(\mathrm{dB})\end{array}$ & $\begin{array}{c}\mathrm{R}_{\text {pot }} \\
(\mathrm{dB})\end{array}$ \\
\hline UL & AP-P & - & $-30,53$ & - \\
\hline UL & AP-M & $\mathrm{db3}$ & $-33,34$ & - \\
\hline UL & DPD-P & - & $-31,39$ & 0,52 \\
\hline UL & DPD-M & db6 & $-32,56$ & 2,71 \\
\hline DL & AP-P & - & $-29,49$ & - \\
\hline DL & AP-M & $\mathrm{db5}$ & $-33,94$ & - \\
\hline DL & DPD-P & - & $-32,41$ & 0,87 \\
\hline DL & DPD-M & $\mathrm{db} 4$ & $-33,03$ & 3,31 \\
\hline
\end{tabular}

Se puede ver como el modelado de AP y DPD mejora con la nueva técnica en todos los casos, llegando a alcanzar $4,45 \quad \mathrm{~dB} \quad \mathrm{y} \quad 1,17 \quad \mathrm{~dB}$ respectivamente. En cuanto a la mejora de la $\mathrm{R}_{\mathrm{pot}}$ alcanza los 2,44 dB.

\section{Conclusiones}

La técnica de modelado propuesta mejora el error tanto en el modelado del AP como en la identificación del DPD, obteniendo mejores resultados para el primero de los dos. Además también es capaz de reducir mejor la ACI respecto al modelo clásico polinomial. En cuanto a la coherencia también se puede ver en la Fig. 2 como es mejor al utilizar el método propuesto.

\section{Referencias}

[1]. D. R. MORGAN, Z. MA, J. KIM, M. G. ZIERDT, and J. PASTALAN. A generalized memory polynomial model for digital predistortion of RF power amplifiers. IEEE Transactions On Signal Processing, 2006, 54(10), 3852-3860.

[2]. L. HONG, G. CHEN, and C. K. CHUI. A FilterBankBased Kalman Filtering Technique for Wavelet Estimation and Decomposition of Random Signals. IEEE Transactions on Circuits \& Sistems: Analog and Digital Signal Processing, 1998, 45(2), 237-241.

[3]. S. R. CHOWDHURY, and D. CHAKRABART. Daubechies Wavelet Decomposition based Baseline wander correction of Trans-Abdominal Maternal ECG. In $6^{\text {th }}$ Int. Conf. on Electrical and Computer Engineering, Piscataway: IEEE, 2010, pp. 630-633.
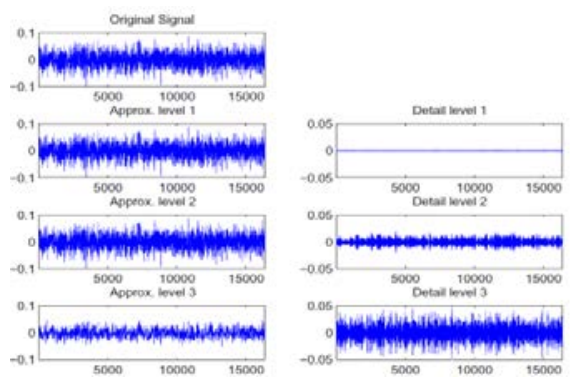

Fig. 1. Descomposición de la señal original en aproximaciones y detalles.
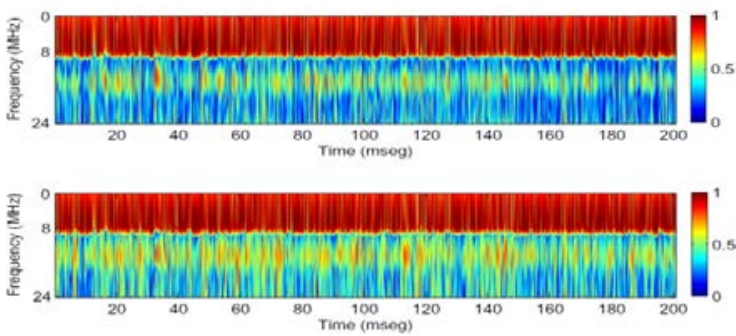

Fig. 2. Coherencia entre la señal de entrada y la señal de salida linealizada: (a) Modelo Polinomial (b) Modelo Multirresolución.

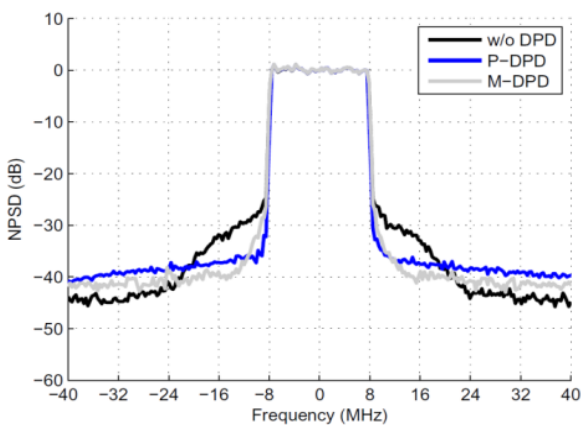

Fig. 3. Densidad Espectral de Potencia Normalizada de la señal Uplink utilizando DPD-P y DPD-M. 